them ; a qualification of this kind must either seem quite baffling, unless its motivation is explained carefully, or it will simply be ignored.

Agaịn, Professor Elandt-Johnson does not point out the very different reliability of the various methods. In theory, the Hardy-Weinberg law should scarcely ever hold in real populations, since there are always disturbing factors. In practice, it almost always holds, with sufficient accuracy for any practical purpose. In contrast, the elegant theory of ascertainment probability for a rare genetic condition must be scarcely ever applicable to real data. For it is based on an assumption that all affected individuals have, independently of one another, the same probability of being a proband (that is, of coming directly to the notice of the investigator, excluding cases found only because they are relatives of some other affected individual). It is very difficult to think of a reasonable and practicable method of sampling with the property that two affected brothers have independent chances of being probands in this sense. (Random sampling is not practicable here.) For example, one commonly used sampling procedure will be through enquiries made to medical practitioners; but brothers, especially if they are young, will often have the same physician. Moreover, failure of this assumption will quite appreciably affect the accuracy of the conclusions obtained. The perfection of the book, in which the subject is mostly presented neatly and tidily in best Sunday dress, also conceals the fact that real life is not always so perfect; populations are heterogeneous, genetic conditions do not always follow Mendelian ratios, estimates of mutation rates are inconsistent, samples are heavily biased, and major theoretical problems remain unsolved or controversial. For these reasons the book must be read with caution and with awareness of reservations. Subject to this, it represents a useful exposition of some of the most important parts of mathematical genetics.

Cedric A. B. SMIth

\section{Liquid Scintillation}

Liquid Scintillation Counting. Edited by A. Dyer. (Symposium at University of Salford, September 1970.) Pp. vii +133. (Heyden: London, New York and Rheiner, July 1971.) £4.60; $\$ 12$.

THe principle of liquid scintillation counting is deceptively simple. Its practice has become remarkably complex. A specimen labelled with a $\beta$-emitter, usually tritium or carbon-14, is introduced into a fluorescent organic solution, and the scintillations induced in the solution by the $\beta$-particles are detected by a pair of photomultipliers, operating in coincidence to minimize background noise. With the development in recent years of many increasingly sophisticated automatic counting instruments, liquid scintillation counting has become established as the basic technique for the radio-assay of biological specimens.

The complexities arise from the variety of biological materials and their physico-chemical properties; the numerous sample preparation techniques and solubilizers which are used to incorporate them into the solution; the many different scintillator solutions which are used, and the intricate sequence of events which constitutes the scintillation process. To quote Dr Kalbhen's concluding remarks at this symposium: "For each variation in solvent, solute and sample preparation techniques, it is necessary to recalibrate the liquid scintillation counter to optimal counting conditions. It has become quite impossible to compare quantitatively counting results from one laboratory with those from another". In spite of these limitations, which may ultimately be overcome by the adoption of standardized sample preparation methods which convert all biological materials into the same combustion products, satisfactory liquid scintillation counting methods have been developed for the routine radio-assay of a very wide range of biological materials.

The thirteen papers in this slim volume were originally presented at a two-day symposium at the University of Salford. Topics discussed include chemiluminescence as a problem and as an analytical tool, the emission spectra of liquid scintillators, the determination of the activities of carbon-14 and electroncaptive nuclides, and a new gelifying agent for specimen incorporation. Three papers are devoted to the use of computer techniques for data processing and quench correction, and two deal with specific biological applications to thyroid cell DNA, enzymes and drug actions. Two papers on scintillations in liquid helium and the design of a liquid helium neutron polarimeter seem strangely out of context. The contents of the volume seem to be too diverse to appeal to anyone who is not already a specialist in the subject. J. B. BIRKS

\section{Nuclear Reactions}

Nuclear Reactions and Nuclear Structure. By P. E. Hodgson. Pp. xii +661 . (Clarendon: Oxford; Oxford University: London, October 1971.) £14.50.

Peter Hodgson is a physicist's physicist, a man poised half way between the theoretical and experimental world, who provides the necessary bridge between the desk bound and bench bound nuclear physicist. Although truly a theoretician, Hodgson still hankers after his experimental beginnings as a research worker and with such a background he is well suited to write a book such as this that aims particularly at the experimental physicist. It is this background that perhaps makes this book the eminently readable book that it is-a rare quality in a volume that is a comprehensive survey of a kinetic subject such as nuclear reaction theory.

Nuclear Reactions and Nuclear Structure comes eight years after the author's first book in this field, The Optical Model of Elastic Scattering, also published by the Clarendon Press. Not unnaturally there is a deal of common ground covered but these sections have been completely revised with references included up to 1971. In 1963, Hodgson treated only the scattering of nucleons but now he turns his attention to the full complexities of nuclear reactions as well without, however, going into details of how gamma ray data can aid in unravelling the details of reaction mechanisms. He says in the introduction that the subject matter covered is a matter of his own taste and interest. It is thus not surprising that in this book we find a most comprehensive and up to date treatment of the optical model and distorted wave treatment of nuclear reactions. It is unfortunate in a way, but certainly not so in another, that such a book as this gets dated very quickly, and one has only to compare The Optical Model of Elastic Scattering and the present volume to realize how fashion has changed in the field of elastic scattering in the past eight years.

What sets Hodgson's book apart is his frequent comparisons between experiment and theory, exemplified by figures taken from published articles. This will be welcomed by the experimental nuclear physicists who often have to suffer books that make no attempt to marry theory and experiment. Hodgson also makes no attempt to involve the reader in mathematical calculations and, as he rightly points out in the introduction, the techniques of mathematical manipulation spread over several chapters would be indigestible to the reader. The experimenter, of course, is more concerned with the implications of how the theoretical calculation fits his results than with the subtleties of Racah algebra.

There is no doubt that all experimental physicists working in the field of nuclear reactions should have, and indeed will want, a copy of this book. It is unfortunate that the price will make it improbable that they can. It is a sign of the times that whereas The Optical Model of Elastic Scattering cost $£ 1.50$ for 211 pages, the present book costs $£ 14.50$ for 661 pages, a somewhat disproportionate increase.

AIUN JONES 